\title{
Competition between Specialist and Generalist Methylotrophic Bacteria for an Intermittent Supply of Methylamine
}

\author{
By J. D. LEGAN,$^{1} \dagger$ J. D. OWENS ${ }^{1 *}$ AND G. A. CHILVERS ${ }^{2}$ \\ ${ }^{1}$ Department of Food Technology, University of Reading, Whiteknights, Reading RG6 2AP, UK \\ ${ }^{2}$ Department of Botany, Australian National University, PO Box 4, Canberra, ACT 2601, \\ Australia
}

(Received 19 June 1986; revised 23 October 1986)

\begin{abstract}
A computer program, METHCOMP, to simulate the competition between an obligately methylotrophic bacterium, a facultatively methylotrophic bacterium, a bacterium able to use methylamine as nitrogen source only, and a non-methylamine-using bacterium for methylamine and glucose in continuous culture with a constant or alternating nutrient supply is described. Competitions between obligate methylotroph B6/2 and facultative methylotroph DT26 in continuous cultures supplied alternately with methylamine and glucose/ammonium were both simulated using experimentally determined growth parameters and investigated in laboratory mixed cultures. The results of the laboratory experiments were in excellent agreement with the simulations. The composition of the mixed population is predicted to vary with imposed dilution rate, with strain B6/2 excluded at dilution rates below $0.0625 \mathrm{~h}^{-1}$ and strain DT26 excluded at dilution rates above $0 \cdot 20 \mathrm{~h}^{-1}$. At intermediate dilution rates both strains coexisted in stable proportions which depended upon the dilution rate. Hence, the use of an alternating nutrient supply may offer a practical approach to the control of the composition of defined, mixed microbial populations.
\end{abstract}

\section{INTRODUCTION}

Mixed microbial populations are the rule in many traditional food and drink fermentations but have been little exploited otherwise, in spite of potential advantages over axenic cultures, such as higher growth rate, higher yield, and greater stability (Harrison, 1978; Larsen et al., 1978). The development of new mixed culture processes is hampered by the absence of rational techniques for culture formulation and for controlling culture composition.

One major aim of this work was to develop an experimental model system to study means of controlling the composition of mixed cultures by alternating the supply of two nutrients so as to favour each of two coexisting bacteria in turn. The system examined was based upon bacterial competition for methylamine, with glucose as the alternating nutrient. The second major aim of the work was to test certain hypotheses about the ecology of methylamine-using bacteria.

Bacteria able to use methylamine under aerobic conditions include at least three main physiological types, namely $(a)$ specialist, obligately methylotrophic bacteria able to use methylamine but not non- $\mathrm{C}_{1}$ compounds as sole carbon and energy source; $(b)$ generalist, facultative methylotrophs able to use methylamine or other compounds singly as sole carbon and energy source; and $(c)$ methazotrophs which cannot use methylamine as sole carbon and energy source but, in the presence of another source of carbon and energy, can use it as sole nitrogen source. The existence of the methazotrophs adds an extra dimension to the model not possible

† Present address: Flour Milling and Baking Research Association, Chorleywood, Rickmansworth, Herts, WD3 5SH, UK. 
with competition for non-nitrogenous organic compounds. A fourth physiological group, $(d)$, of non-methylamine-using heterotrophs adds a final level of complexity to the model. The biochemistry of methylotrophy and methazotrophy is relatively well understood (Zatman, 1981 ; Anthony, 1982; Large \& Green, 1984). Thus, ultimately, it may be possible to relate the ecology of these organisms to the possession of specific biochemical attributes.

A generalist microbe, with a flexible metabolism capable of using many different substrates, might use different substrates one at a time or use them simultaneously. Both of these behaviours have been described for facultatively chemolithotrophic thiobacilli (Gottschal et al., 1981 $a, b$; Kuenen \& Gottschal, 1982). Adaptation of certain photosynthetic bacteria to intermittent illumination was demonstrated by Van Gemerden (1974).

Chemostat enrichment cultures under methylamine-nitrogen limitation with excess glucose invariably yield methazotrophs and not facultative methylamine-using methylotrophs $(\mathrm{J}$. D. Owens, unpublished observations). Hence, it would seem that the strategy of simultaneously using methylamine and glucose is denied to facultatively methylamine-using bacteria. We suggest, therefore, that facultatively methylamine-using bacteria benefit from their ability to use methylamine only when the availability of methylamine is intermittent. A major aim of the present work was to test this hypothesis.

This study was designed from the first to include both computer simulation and experimental testing of the predictions of the simulations. A number of studies combining mathematical modelling and experimental studies on continuous mixed cultures in constant environments (Gottschal \& Thingstad, 1982; Kemp et al., 1983) or on continuous axenic cultures in varying environments (Pickett, 1982) have been published. However, apart from a report on the effect of pH oscillations on a mixed culture (Davison \& Stephanopoulos, 1986), studies of mixed cultures in varying environments seem to have been done either only in the laboratory (Van Gemerden, 1974; Turpin \& Harrison, 1979; Gottschal et al., 1981 $a, b$ ) or by mathematical modelling alone (Stewart \& Levin, 1973; Klei et al., 1975). In the present work the competition between an obligate methylotroph and a facultative methylotroph in a continuous culture alternately supplied with methylamine and glucose as growth-rate-limiting nutrients was studied, both with computer simulations and in laboratory cultures. Only two strains were used for these initial studies in order to keep the system as simple as possible and to facilitate the experimental studies.

\section{METHODS}

Bacterial strains. The strains used derive from isolates obtained from water from a pond in Sydney, Australia. Strain B6/2 is a non-adhesive variant (Legan, 1985; Legan \& Owens, 1985) of an original isolate obtained from a continuous enrichment culture on a mineral salts $/$ vitamins $/ 0.3 \mathrm{mmol}^{-1}$ methylamine medium supplied at a dilution rate of $0 \cdot 14 \mathrm{~h}^{-1}$. It is a Gram-negative, rod-shaped, non-motile, obligately aerobic organism which grows well on methylamine and methanol and extremely slowly on glucose (doubling time $>30 \mathrm{~h}$ ) but not on a range of other compounds tested. It is thus a near obligate methylotroph according to Zatman's (1981) nomenclature but is referred to as an obligate methylotroph for the purposes of this paper.

Strain DT26 was isolated by Bicknell (1978) by a direct plating technique as described by Bicknell \& Owens (1980). It is a Gram-negative, rod-shaped, motile, facultative methylotroph able to grow on methylamine, dimethylamine, trimethylamine, trimethylamine oxide and a wide variety of non- $\mathrm{C}_{1}$ organic compounds, including glucose.

These two strains were selected from those available because of the low proportion of flocculated cells and the absence of wall growth in continuous cultures.

Culture media. Continuous cultures were grown in mineral base $\mathrm{C}$ (a mineral salts/EDTA/trace metals mixture, pH 6.8; Legan \& Owens, 1985) with filter-sterilized glucose added aseptically to a final concentration of $0.005 \mathrm{M}$ or in mineral base $\mathrm{C}-\mathrm{N}$ (base $\mathrm{C}$ with the nitrogen component omitted) with filter-sterilized methylamine added to a final concentration of $0.03 \mathrm{M}$.

Static flask cultures were grown on mineral base E (a mineral salts/phosphate buffer/EDTA/trace metals mixture, pH 6.8; Owens \& Keddie, 1969) with aseptically added glucose at a final concentration of $0.005 \mathrm{M}$ or in base $\mathrm{E}-\mathrm{N}$ (base $\mathrm{E}$ with the nitrogen component omitted) with aseptically added methylamine at a final concentration of $0.03 \mathrm{M}$.

Agar media used were: medium MA (base E mineral salts mixture, 11; methylamine. $\mathrm{HCl}, 2.025 \mathrm{~g}$; purified agar, Oxoid L28, $15 \mathrm{~g} ; \mathrm{pH} \mathrm{6.8);} \mathrm{medium} \mathrm{GA} \mathrm{(base} \mathrm{E,} 11$; glucose, $0.9 \mathrm{~g}$; purified agar, $15 \mathrm{~g} ; \mathrm{pH} 6.8$ ); and medium 
YMGA (base E, 11 ; yeast extract Oxoid L21, $1 \mathrm{~g}$; methylamine. $\mathrm{HCl}, 2.025 \mathrm{~g}$; glucose, $0.9 \mathrm{~g}$; purified agar, $15 \mathrm{~g}$; pH 6.8). They were prepared by aseptically mixing equal amounts of sterile double-strength base $E$ and doublestrength distilled water agar with appropriate amounts of filter-sterilized stock solutions of the other ingredients made at 100 times the final concentrations in the media.

Continuous culture apparatus. Continuous cultures were grown in an LH Fermentation 500 series III fermenter. Medium was supplied from two reservoirs by two Gilson Minipuls 2 pumps connected to a purpose-built two-way time switch which allowed the reservoirs to be alternated at preset intervals. The time switch was constructed from two process timers (Crouzet 88 276).

The culture volume was maintained at $670 \mathrm{ml}$ in a 11 Pyrex glass vessel by an internal weir. Temperature was controlled at $25^{\circ} \mathrm{C}$ by means of an electric immersion heater and a cooling finger, through which was passed a carefully regulated flow of cold water from a constant-head device. Cultures were mixed by stirring at 750 r.p.m and aerated with $400 \mathrm{ml}$ sterile air $\mathrm{min}^{-1}$. The dissolved oxygen tension in cultures was monitored to ensure that it never fell below $50 \%$ of the saturation value in equilibrium with air. Measurement of respiration rates of cultures in a Rank oxygen electrode respirometer, in the presence of excess substrate, showed that rate-limiting concentrations of dissolved oxygen were all below $25 \%$ of saturation value in equilibrium with air. Gas was exhausted from the culture vessel through a water cooled condenser. Automatic titration with $0 \cdot 1 \mathrm{M}-\mathrm{NaOH}$ or $0 \cdot 1 \mathrm{M}-\mathrm{HCl}$ maintained the culture at a $\mathrm{pH}$ value of $6 \cdot 8 \pm 0 \cdot 05$.

Operation of mixed cultures with an alternating nutrient supply. These experiments were done under the following conditions.

(1) Two bacterial strains were used, obligate methylotroph B6/2 and facultative methylotroph DT26.

(2) To start cultures, $300 \mathrm{ml}$ of a late exponential-phase shake-flask culture of each strain was injected into the empty culture vessel. The culture volume was made up to the weir level with equal volumes of medium from each reservoir. The medium pumps were started and the timer was set to supply medium alternately from each reservoir for the desired periods.

(3) Substrate concentrations in the reservoirs were $(\mathrm{mM})$ : reservoir I: methylamine, 30; glucose, $0 ; \mathrm{NH}_{4}^{+}, 0$; reservoir II: methylamine, 0 ; glucose, $5 ; \mathrm{NH}_{4}^{+}, 2$. The concentrations of glucose and methylamine were chosen so that the biomass concentration of facultative methylotroph DT26 would be the same on each.

The substrates were supplied for much shorter periods than seem likely to be encountered in a natural environment for three reasons: $(a)$ the amplitude of oscillation in the population composition is reduced, which makes the stable state easier to recognize; $(b)$ the probability of a growth lag on switching substrates in laboratory culture is reduced; and $(c)$ the selective pressure for constitutive mutants, which occurs with pulsed feeding and long starvation periods (Ingraham et al., 1983), is reduced. The relatively rapid alternation of nutrients used here must exert some selective pressure for variants adapted to these conditions. However, apart from some tendency to the production of smaller colonies on agar media, no substantial changes in the properties of the microbes were observed over the periods of the present experiments. Thus two potential sources of disagreement between results of simulated and laboratory cultures were largely removed.

(4) Methylamine and glucose $/ \mathrm{NH}_{4}^{+}$were supplied alternately for periods of $0 \cdot 1$ of a residence time $(0 \cdot 1 / D)$. The absolute length of time for which each substrate was supplied, therefore, decreased as the dilution rate increased. The medium pumps were set so that the flow rate from each reservoir was equal.

(5) Under some conditions. mixed populations can take a very long time to become completely stable. Furthermore, the population composition changes only very slowly as it approaches stable conditions, making it very difficult to tell whether or not stability has been attained. Cultures were therefore run until the results of computer simulations predicted that the most numerous organism was within $5 \%$ of its stable value. Generally, a culture was run for at least four residence times longer than this predicted time and the population was monitored periodically, including at least once every residence time for the last four residence times. Provided that the final four samples showed a reasonably stable population composition the culture was presumed to have reached stable state and the experiment was ended.

(6) Samples were taken from continuous cultures at intervals determined by the dilution rate. Cultures were sampled more frequently during the early stages, when the population composition changes relatively rapidly, than in the later stages. The shortest period between samples was 0.25 residence time $\left(\right.$ at $\left.D=0.04 \mathrm{~h}^{-1}\right)$ and the longest period was two residence times (at $D=0.20 \mathrm{~h}^{-1}$ ).

Enumeration and identification of micro-organisms. Cells of strains B6/2 and DT26 in mixed cultures were enumerated and differentiated by spreading two portions $(0.1 \mathrm{ml})$ of appropriate dilutions over plates of medium MA, medium GA and medium YMGA. Colonies on medium MA were counted after 2 and $4 \mathrm{~d}$ incubation at $25^{\circ} \mathrm{C}$. Colonies appearing after $2 \mathrm{~d}$ were those of obligate methylotroph B6/2 and colonies appearing after $4 \mathrm{~d}$ were those of facultative methylotroph DT26. Colonies on media GA and YMGA were counted after $4 \mathrm{~d}$, large (approx. $3 \mathrm{~mm}$ diameter) and small (approx. $1 \mathrm{~mm}$ diameter) colonies being recorded separately. Large colonies were those of strain DT 26 and small colonies were those of strain B6/2. The viable counts of each strain were normally taken as those determined on medium YMGA. However, if these differed appreciably from the results obtained on the other two media the means of the viable counts on medium MA and medium GA were used. 
Read initial values and kinetics data for:

four bacteria (obligate methylotroph, facultative methylotroph, methazotroph, heterotroph unable to use methylamine)

three nutrients (methylamine, glucose, ammonium)

two nutrient supply reservoirs (e.g. reservoir I, methylamine; reservoir II, glucose and ammonium)

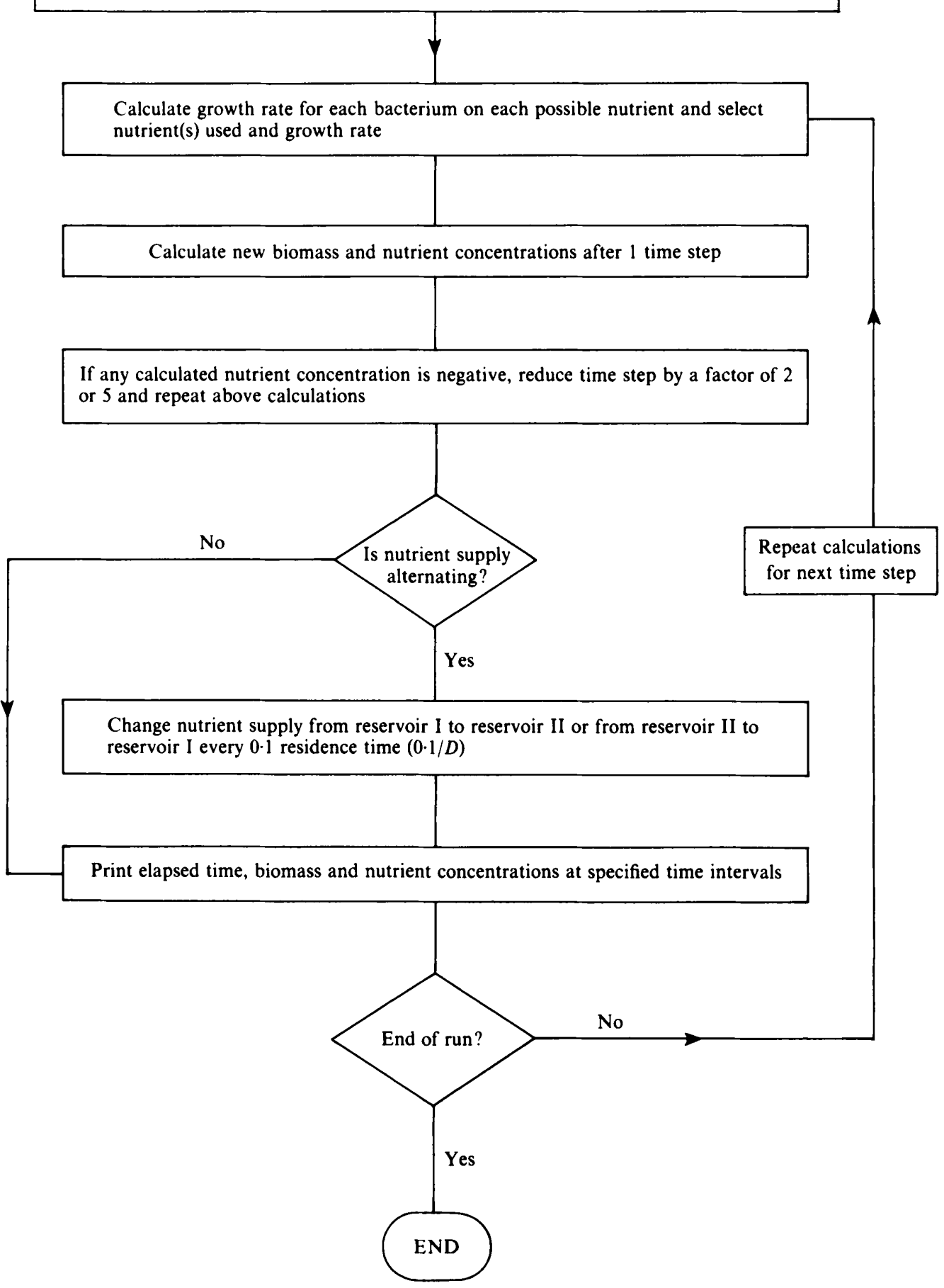


METHCOMP computer simulation program. Simulations were done with a Commodore model 8096 or 4032 computer, using the program METHCOMP31EPSON or METHCOMP33CBM (versions of the same program adapted for use with different printers). A flow diagram of the program is shown in Fig. 1. The program is written in BASIC and requires approximately $9.2 \mathrm{~kb}$ RAM. A listing is available on request.

The METHCOMP program simulates the competition of four different bacterial types for three substrates in continuous culture with a constant or alternating nutrient supply. Specifically, it simulates the competition for methylamine, glucose and ammonium of an obligately methylamine-using bacterium, a facultatively methylamine-using bacterium, a methazotroph, and a non-methylamine-using heterotroph. Any combination of nutrients can be supplied constantly from one reservoir to simulate the operation of a normal chemostat. Alternatively, a chemostat fed alternately from two nutrient reservoirs may be simulated. The nutrient content and on/off time of each reservoir can be set independently. The program incorporates the following assumptions and procedures.

(1) The culture is perfectly homogeneous.

(2) Bacterial growth rate, $\mu$, is determined by the concentration of a single growth-rate-limiting nutrient according to the Monod equation:

$$
\mu=\frac{\mu_{\mathrm{m}} s}{K_{\mathrm{s}}+s}
$$

where $\mu_{\mathrm{m}}$ is the maximum specific growth rate, $K$ is the substrate saturation constant, and $s$ is the concentration of growth-rate-limiting nutrient in the culture. Double substrate-limited growth is excluded (Bader, 1982).

(3) Growth commences with no lag phase.

(4) Yield coefficients are constant for all growth rates.

(5) A bacterial strain uses only one carbon and one nitrogen source at a time. For example, the facultative methylotroph grows either on methylamine as sole carbon and nitrogen source or on glucose/ $\mathrm{NH}_{4}^{+}$but does not simultaneously use methylamine and glucose. This simplifying assumption is probably valid when one or both nutrients are present at relatively high concentrations but may not hold when both are at low concentrations. Nevertheless, this assumption seems preferable to the arbitrary estimation of the proportion of each nutrient used.

(6) Each bacterial strain uses the carbon source and nitrogen source that supports the highest growth rate with the prevailing nutrient concentrations. The procedure to determine which nutrients are used by a particular bacterium, and the growth rate supported, is as follows: (i) growth rates are calculated for each possible nutrient; (ii) the carbon source used is that which supports the highest growth rate; (iii) the nitrogen source used is that which supports the highest growth rate; and (iv) the growth rate of the bacterium is set equal to the lower of the rates on the selected carbon and nitrogen sources.

(7) Bacterial growth is computed by making successive linear approximations of the exponential growth equation over small periods of time (the time step). Thus in continuous culture, after one time step, $\mathrm{d} t$, the biomass concentration, $x_{t+1}$, of a bacterium with growth rate $\mu$ is given by:

$$
x_{t+1}=x_{t}+x_{t} \mathrm{~d} t(\mu-D)
$$

where $x_{t}$ is biomass concentration at the start of the time interval and $D$ is culture dilution rate. The substrate concentration, $s_{t+1}$, after the time step is given by:

$$
s_{t+1}=s_{\mathrm{t}}+D \mathrm{~d} t\left(S-s_{t}\right)-\Sigma \mu x_{t} \mathrm{~d} t / Y
$$

where $s_{t}$ is the concentration of nutrient at the start of the time interval, $S$ is the concentration of nutrient in the reservoir, $Y$ is the yield coefficient of bacterial biomass per unit of nutrient, and $\Sigma \mu x_{t} \mathrm{~d} t / Y$ is the sum of the nutrient used by the different microbes for growth.

(8) Growth on methylamine as carbon and nitrogen source results in excretion of $\mathrm{NH}_{4}^{+}$. The concentration of $\mathrm{NH}_{4}^{+}$produced, $c$, by each bacterium for one time step is given by:

$$
c=\mu x_{t} \mathrm{~d} t\left[\left(1 / Y_{\mathrm{C}}\right)-\left(1 / Y_{\mathrm{N}}\right)\right]
$$

where $Y_{\mathrm{C}}$ is the yield coefficient with respect to carbon source and $Y_{\mathrm{N}}$ is the yield coefficient for nitrogen source.

(9) When substrates are supplied alternately, a true steady state is not achieved. The population does, however, reach a state where the composition cycles repeatedly through the same values. This will be termed a stable state.

Fig. 1. Flow diagram of the METHCOMP computer program for simulating the competition of four bacteria for three nutrients in continuous culture with a constant or an alternating nutrient supply. Equations and procedures used are given in the text. 
Table 1. Growth parameters used in computer simulations of mixed continuous cultures of obligate methylotroph B6/2 and facultative methylotroph DT26

Maximum specific growth rates were experimentally determined. Substrate saturation constants were experimentally determined for carbon source and the associated nitrogen source (i.e. $\mathrm{NH}_{4}^{+}$) was assigned the same value. Yield constants were experimentally determined at $50 \%$ of maximum growth rate for carbon sources. Yield constant on nitrogen source is the theoretical value assuming that approximately $12 \%$ of cell dry weight is nitrogen. Cultures simulated using these values were all carbon limited. Data from Legan \& Owens (1987).

$\begin{array}{llcccc}\text { Strain } & \text { Substrate } & \begin{array}{c}\text { Max. specific } \\ \text { growth rate } \\ \left(\mathrm{h}^{-1}\right)\end{array} & \begin{array}{c}\text { Substrate } \\ \text { saturation } \\ \text { constant } \\ \left(\mu \mathrm{mol} \mathrm{I}^{-1}\right)\end{array} & \begin{array}{c}\text { C-source } \\ \text { [g dry biomass (mol } \\ \text { substrate) }\end{array} & \text { N-source } \\ \text { B6/2 } & \text { Methylamine } & 0 \cdot 33 & 36 & 21 \cdot 2 & 120 \\ \text { DT26 } & \text { Methylamine } & 0 \cdot 09 & 5 \cdot 1 & 15 \cdot 6 & 120 \\ \text { DT26 } & \text { Glucose } & 0 \cdot 20 & 2 \cdot 5 & 94 & \text { NA } \\ \text { DT26 } & \text { NH4 }_{4}^{+} & 0 \cdot 20 & 2 \cdot 5 & \text { NA } & 120\end{array}$

NA, Not applicable.

Simulated competitions between obligate methylotroph B6/2 and facultative methylotroph DT26 in alternating environments. The METHCOMP program was used to simulate the competition between these two strains under the following conditions.

(1) Experimentally determined values of growth parameters (Table 1) were used.

(2) The reservoir nutrient concentrations were the same as those used in the laboratory cultures.

(3) Substrate was supplied from each reservoir alternately for $0 \cdot 1$ residence time.

(4) Initial biomass concentrations were $150 \mathrm{mg} \mathrm{l}^{-1}$ of each strain.

(5) The initial concentration of $\mathrm{NH}_{4}^{+}$in the culture was $2 \mathrm{mM}$ and the concentrations of glucose and methylamine were one-fifth of the reservoir concentrations. This reduced the amount by which the culture density overshot its maximum value, and hence the time taken to reach the stable state. It also fairly realistically described the way in which experimental mixed cultures were inoculated.

(6) The simulation was run until a mixed culture in a stable state was obtained. A stable state was recognized when the population composition cycled through the same values for not less than four residence times, or when the concentration of one of the strains fell to zero. Final values were not necessarily reached but the rate of change of the population composition became too low to be recognized.

\section{RESULTS AND DISCUSSION}

\section{Relationships between specific growth rate and substrate concentration}

The growth rate versus methylamine concentration curves of obligate methylotroph $\mathrm{B} 6 / 2$ and facultative methylotroph DT26 cross at a growth rate of $0.05 \mathrm{~h}^{-1}$ (Legan \& Owens, 1987). In continuous cultures operated with a constant methylamine supply, strain B6/2 is thus expected to predominate at dilution rates above $0.05 \mathrm{~h}^{-1}$. At dilution rates below $0.05 \mathrm{~h}^{-1}$ strain DT26 is expected to predominate.

\section{Simulated competitions between obligate methylotroph B6/2 and facultative methylotroph DT26 supplied alternately with methylamine and glucose}

Changes in population composition during simulated competitions. Examples of computersimulated competitions between obligate methylotroph B6/2 and facultative methylotroph DT26 are shown in Fig. 2. Some of the simulations were very slow to reach a stable state. At a dilution rate of $0.08 \mathrm{~h}^{-1}$ it took more than $350 \mathrm{~h}$ (equivalent to 28 residence times), although the eventual outcome was evident much earlier (Fig. $2 a$ ). Simulations at higher dilution rates reached stability more rapidly. At a dilution rate of $0.16 \mathrm{~h}^{-1}$ stability was reached in less than $100 \mathrm{~h}$ (equivalent to 16 residence times; Fig. $2 b$ ).

The relatively long times required for these simulations, which do not, of course, suffer from the sampling and enumeration errors inherent in laboratory experiments, draw attention to the 


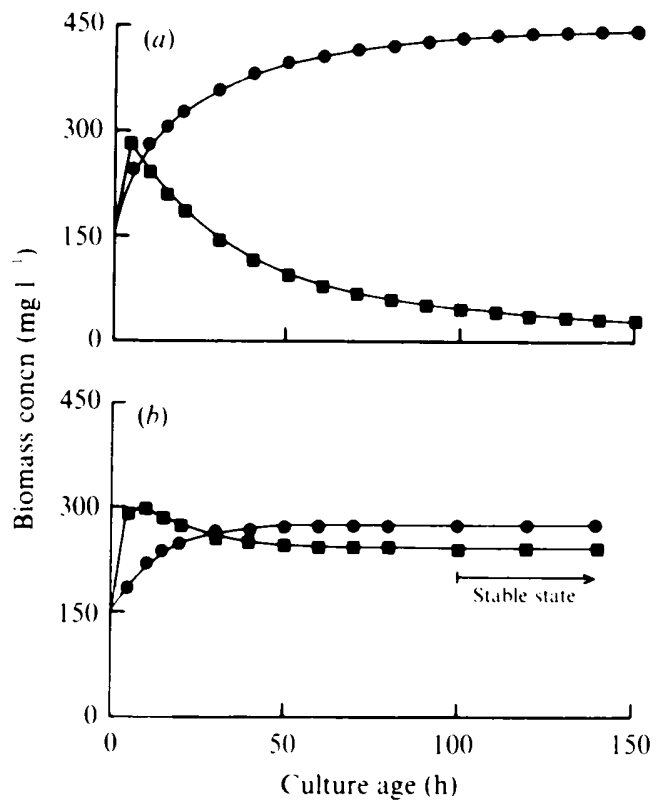

Fig. 2. Simulated competitions between obligate methylotroph B6/2 ( $\square$ ) and facultative methylotroph DT26 (O). Methylamine and glucose were supplied alternately for periods of $0 \cdot 1$ residence time. (a) Dilution rate $=0.08 \mathrm{~h}^{-1} ;(b)$ dilution rate $=0 \cdot 16 \mathrm{~h}^{-1}$. Experimentally determined growth parameters were used. Concentrations (mM) of methylamine, glucose and ammonium respectively were : reservoir I, 30, 0, 0; reservoir II, 0, 5, 2. Simulation was done using the METHCOMP program.

potential difficulties of doing such experiments with real cultures, and to the need for such experiments to be operated for long enough to allow final stable states to be approached even if not reached. Kuenen \& Gottschal (1982), as a result of a later computer simulation study, suggested that some inconsistencies between earlier experimental data and theoretical expectations were probably due to not running the experimental cultures for long enough. Their observations, and those made here, indicate the value of doing computer simulations before embarking on laboratory experiments that may not be capable of yielding meaningful results in practical time periods.

Composition of stable-state populations. The stable population compositions of the simulated cultures are shown in Fig. 3. The population composition changed gradually with dilution rate. At dilution rates below $0.0625 \mathrm{~h}^{-1}$ the culture was dominated by facultative methylotroph DT26 and obligate methylotroph B6/2 was excluded. The proportion of obligate methylotroph B6/2 in the mixed continuous culture increased with increasing dilution rate. At a dilution rate of $0 \cdot 16 \mathrm{~h}^{-1}$ the population contained approximately $50 \%$ of each strain.

These results may be explained by considering the balance of advantages between the two strains. Facultative methylotroph DT26 is benefited at all dilution rates by the supply of glucose. Obligate methylotroph B6/2 is benefited at all dilution rates above $0.05 \mathrm{~h}^{-1}$ by the supply of methylamine but the size of that advantage increases with increase in the dilution rate. At dilution rates below $0.05 \mathrm{~h}^{-1}$ strain B6/2 grows more slowly than strain DT26 on methylamine and would, therefore, be excluded even in the absence of an intermittent glucose supply. In fact it was excluded at dilution rates below $0.0625 \mathrm{~h}^{-1}$, since the advantage of a slightly faster growth rate on methylamine was outweighed by the ability of strain DT26 to grow on the glucose. At higher dilution rates the growth rate advantage on methylamine of strain B6/2 over strain DT26 increases, and its proportion in the population increased, reaching nearly $50 \%$ at a dilution rate of $0 \cdot 16 \mathrm{~h}^{-1}$.

The maximum specific growth rate of facultative methylotroph DT26 on glucose is $0.20 \mathrm{~h}^{-1}$ and at dilution rates above this it is excluded from the culture. However, at dilution rates only 


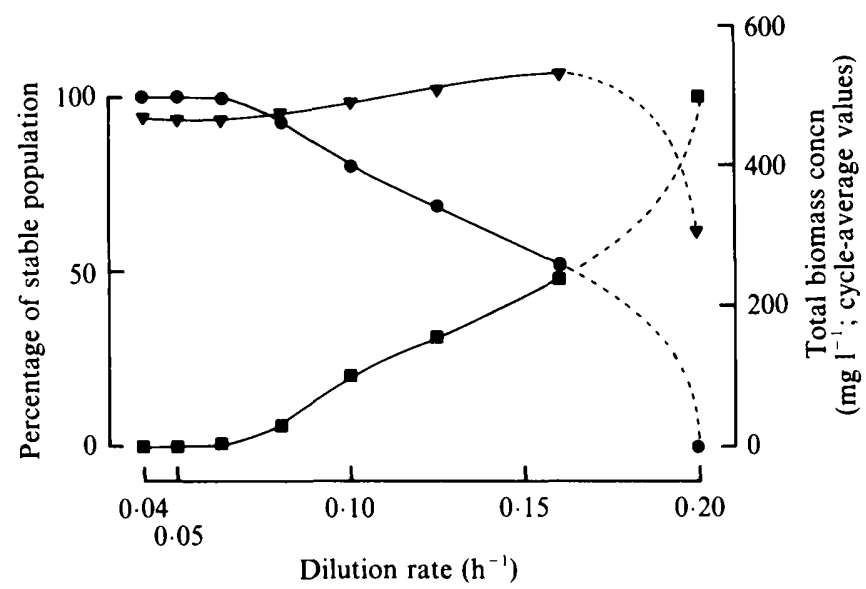

Fig. 3. Biomass concentration $(\nabla)$ and population composition of stable mixed cultures of obligate methylotroph B6/2 ( $\square$ ) and facultative methylotroph DT26 (O) at different dilution rates. Simulated using experimentally determined growth parameters. Concentrations (mM) of methylamine, glucose and ammonium respectively were: reservoir $\mathrm{I}, 30,0,0$; reservoir $\mathrm{II}, 0,5,2$. Glucose/ammonium and methylamine were supplied alternately for periods of 0.1 residence time. Initial concentrations of both strains were $150 \mathrm{mg} \mathrm{l}^{-1}$. Simulation was done using the METHCOMP program.

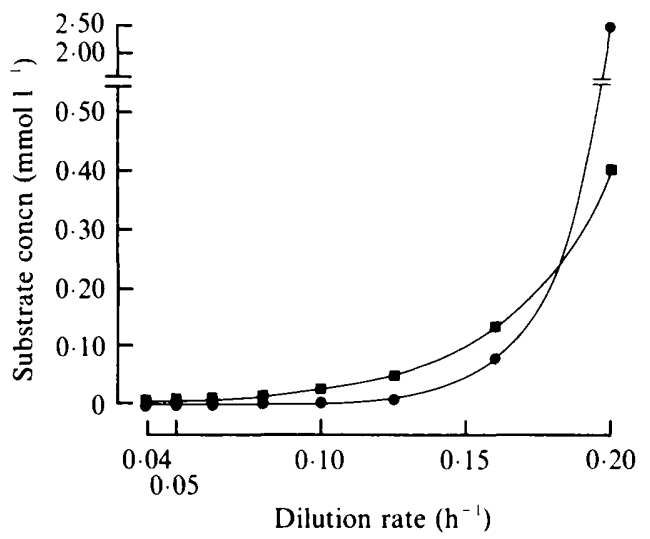

Fig. 4. Cycle average glucose $(O)$ and methylamine $(\square)$ concentrations in stable mixed cultures of obiigate methylotroph B6/2 and facultative methylotroph DT26 at different dilution rates. Simulated using experimentally determined growth parameters. Concentrations (mM) of methylamine, glucose and ammonium respectively were: reservoir $\mathrm{I}, 30,0,0$; reservoir $\mathrm{II}, 0,5,2$. Methylamine and glucose/ammonium were supplied alternately for periods of 0.1 residence time. Initial concentrations of both strains were $150 \mathrm{mg} \mathrm{l}^{-1}$. Simulation was done using the METHCOMP program.

very slightly below $0.20 \mathrm{~h}^{-1}$, it is possible to achieve a stable state containing a very low proportion of strain DT26. This occurs because, at such low population densities, nearly all the glucose is unused and this allows strain DT26 to grow continuously at a rate almost equal to its maximum specific growth rate.

Efficiency of substrate utilization. At dilution rates up to $0 \cdot 16 \mathrm{~h}^{-1}$, substrate utilization is almost complete (Fig. 4) and this is reflected in the high total biomass concentration (Fig. 3). At a dilution rate of $0.20 \mathrm{~h}^{-1}$, virtually all the methylamine is consumed but, because the population of strain DT26 is then virtually zero, nearly all of the glucose is unused. Hence the glucose concentration in the culture comes to a nearly constant value of about half the glucose 


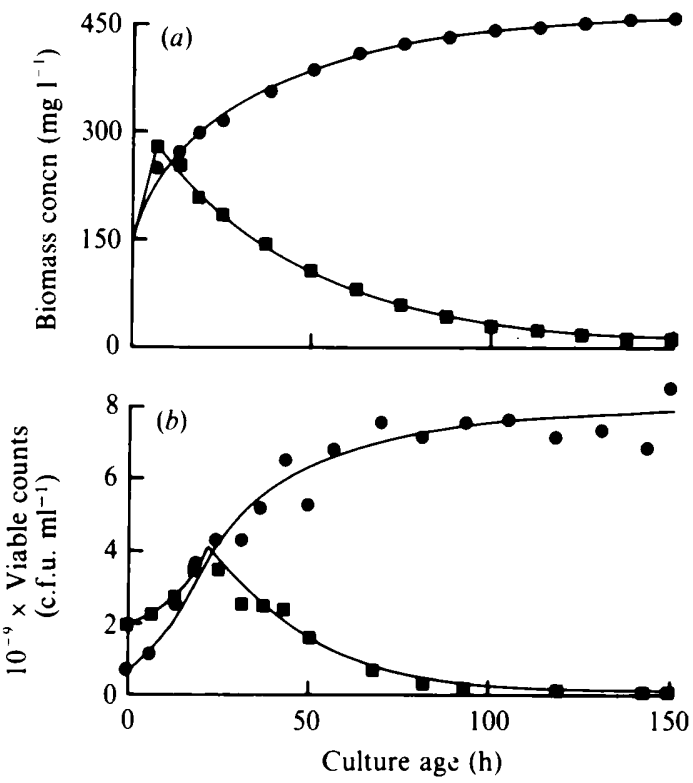

Fig. 5. Simulated $(a)$ and laboratory $(b)$ mixed continuous cultures of obligate methylotroph B6/2 ( $\square$ ) and facultative methylotroph DT26 (O), supplied alternately with glucose and methylamine for $2.5 \mathrm{~h}$ periods at a dilution rate of $0.04 \mathrm{~h}^{-1}$. Concentrations (mM) of methylamine, glucose and ammonium respectively were: reservoir I, 30, 0,0 ; reservoir II, 0,5 , 2 . Simulations used experimentally determined growth parameters and were done using the METHCOMP program.

concentration in reservoir II. Unfortunately it was not possible to simulate dilution rates between 0.16 and $0.20 \mathrm{~h}^{-1}$ and maintain a reservoir alternation period of 0.1 residence time because the program requires that the time step is a common denominator to both the dilution rate and the reservoir alternation period.

Competitions between obligate methylotroph B6/2 and facultative methylotroph DT26 in laboratory continuous cultures alternately supplied with methylamine and glucose/ammonium

Fig. 5 shows the population changes during competitions in both simulated and laboratory continuous cultures alternately supplied with methylamine and glucose/ammonium at a dilution rate of $0.04 \mathrm{~h}^{-1}$. It is evident that the changes predicted by the simulation closely mimic those in the laboratory cultures.

Similar experiments were done at a number of other dilution rates and the final population compositions were compared with the predicted values. Excellent agreement between predicted and observed populations was seen at four dilution rates $\left(0 \cdot 04,0 \cdot 10,0 \cdot 16\right.$ and $0.20 \mathrm{~h}^{-1}$; Table 2) spanning the complete range used in simulation experiments.

Cyclical changes in optical density of cultures with the same period as the substrate alternation were observed but they were small, as expected, since the largest oscillation in the total biomass concentration predicted by the simulation studies was less than $3 \%$ of the mean.

Cyclical changes in dissolved oxygen tension with the same period as the substrate alternation were also observed. These began when the substrate concentrations had fallen to growth-ratelimiting concentrations, but before stable state was reached, and continued for as long as the substrate concentrations remained growth-rate-limiting. The dissolved oxygen tension was lower when the culture was supplied with methylamine than when it was supplied with glucose because, for a given cell population growing at a constant rate, more oxygen is required for the assimilation of methylamine than for that of glucose.

As the age of the continuous cultures increased, the size of colonies of both strains on agar media decreased. This was seen with each continuous culture assessed by colony counts on agar 
Table 2. Compositions of microbial populations in laboratory and computer-simulated mixed continuous cultures of obligate methylotroph B6/2 and facultative methylotroph DT26 supplied alternately with methylamine and glucose/ammonium at different dilution rates

Concentrations (mM) of methylamine, glucose and ammonium respectively were: reservoir I, 30, 0, 0; reservoir II, 0, 5, 2. Methylamine and glucose/ammonium were supplied alternately for periods of $0 \cdot 1$ residence time. Simulations used experimentally determined growth parameters and were done with the METHCOMP program. Results for laboratory cultures are for cultures operated for 5.4 volume changes at $D=0.04 \mathrm{~h}^{-1}, 12$ volume changes at $D=0.10 \mathrm{~h}^{-1}, 14.4$ volume changes at $D=0.16 \mathrm{~h}^{-1}$, and 18 volume changes at $D=0 \cdot 20 \mathrm{~h}^{-1}$.

\begin{tabular}{ccccccc}
$\begin{array}{c}\text { Dilution } \\
\text { rate } \\
\left(\mathrm{h}^{-1}\right)\end{array}$ & $\overbrace{\text { B6/2 }}^{\text {Observed }}$ & DT26 & $\overbrace{\text { B6/2 }}^{\text {Population composition (\%) }}$ & DT26 & & $\overbrace{\text { Observed }}^{\text {Predicted }} \begin{array}{c}\text { Potal biomass } \\
\text { concn }\left(\mathrm{mg} \mathrm{I}^{-1}\right)\end{array}$ \\
$0 \cdot 04$ & 1 & 99 & 0 & 100 & ND & 470 \\
$0 \cdot 10$ & 32 & 68 & 20 & 80 & 470 & 490 \\
$0 \cdot 16$ & 54 & 46 & 48 & 52 & 510 & 530 \\
$0 \cdot 20$ & 96 & 4 & 99 & 1 & 290 & 310 \\
\multicolumn{5}{c}{ ND, Not determined. }
\end{tabular}

media. Changes in strain characteristics leading to reduced colony size, or even complete loss of ability to grow, following transfer from continuous culture to media with relatively high substrate concentrations have been observed by others (Postgate \& Hunter, 1964; Straškbová, 1983; Höfle, 1983), but the physiological mechanisms underlying these observations have not been elucidated.

At no time did the reduction in colony size make it difficult to distinguish between colonies of strains B6/2 and DT26 on media YMGA and GA after $4 \mathrm{~d}$. However, it was difficult to distinguish between the strains on medium MA inoculated from a long-running continuous culture. Colonies of strain B6/2 were easily visible by eye after $2 \mathrm{~d}$ incubation when medium MA was inoculated with material from a young culture, but with material from a continuous culture older than about $100 \mathrm{~h}$ they were quite difficult to see. Extending the incubation time to $3 \mathrm{~d}$ allowed clearly visible colonies of strains B6/2 and DT26 to develop, but then colonies of the two strains were indistinguishable. This could present practical problems in the assessment of threeor four-strain mixed cultures and might impose an upper limit on the time for which the composition of such cultures could be assessed by these methods.

\section{Validity of computer simulation model}

The similarities between the observed changes in population composition in the laboratory mixed cultures and those predicted by computer simulation (Fig. 5), and the closeness of the experimentally observed final population compositions and the predicted ones (Table 2), provide good evidence both that the experimentally determined growth parameters used in the simulations are accurate and that the assumptions in the computer simulation model are acceptable. In particular, they suggest that the following effects, known to greatly affect the final population composition in mixed continuous cultures but not included in the model, did not occur to a significant extent: (1) growth lags when substrates are changed in a culture with an alternating substrate supply (Gottschal et al., 1981 b); (2) inhomogeneities in the culture due to flocculation or wall growth (Ratnam et al., 1982; Baltzis \& Fredrickson, 1983; Chao \& Ramsdell, 1985); and (3) interactions between strains other than competition for substrate (Fredrickson, 1977; De Freitas \& Fredrickson, 1978; Kuenen \& Gottschal, 1982).

Simulated competitions in cultures supplied alternately with methylamine and glucose between bacteria with arbitrarily assigned growth parameters

Maximum specific growth rates of microbes are relatively easily measured but the determination of $K_{\mathrm{s}}$ values is much more difficult. It is, therefore, tempting to do competition 


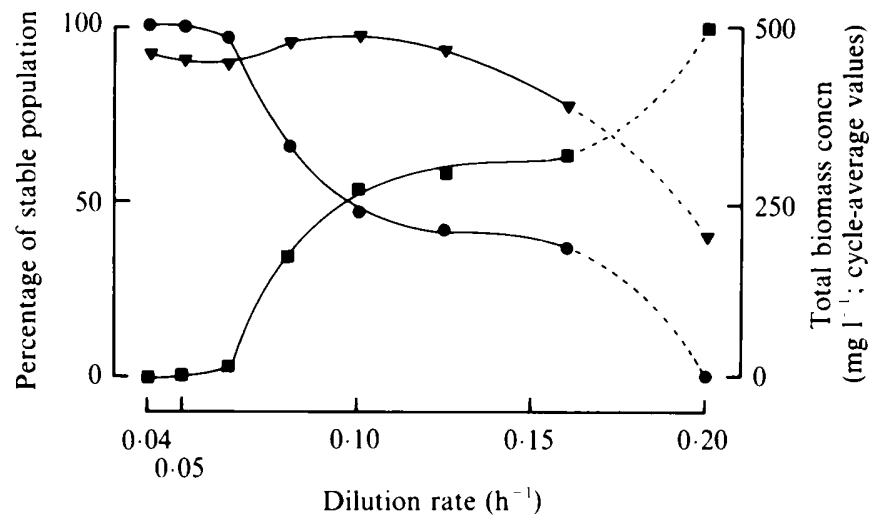

Fig. 6. Biomass concentration $(\nabla)$ and population composition of stable mixed cultures at different dilution rates of hypothetical strains similar to obligate methylotroph $B 6 / 2(\square)$ and facultative methylotroph DT26 (O) but having substrate saturation constants 100 times greater. Concentrations (mM) of methylamine, glucose and ammonium respectively were: reservoir I, 30, 0, 0; reservoir II, 0, 5, 2. Glucose/ammonium and methylamine were supplied alternately for periods of $0 \cdot 1$ residence time. Initial concentrations of both strains were $150 \mathrm{mg} \mathrm{l}^{-1}$. Simulation was done using the METHCOMP program.

simulations using arbitrarily assigned $K_{\mathrm{s}}$ values on the assumption that the outcome of the competitions will be unaltered if the ratio of the $K_{\mathrm{s}}$ values used is similar to the ratio of the true $K_{\mathrm{s}}$ values (e.g. Gottschal \& Thingstad, 1982). The simulations done using experimentally determined growth parameters (Fig. 3) were repeated with the $K_{\mathrm{s}}$ values increased 100 -fold. The results are shown in Fig. 6. The program ran up to 100 times faster and the trends in the two sets of data are similar. Quantitatively, however, the results are quite different except at the extremes of the range $\left(D<0.0625 \mathrm{~h}^{-1}\right.$ and $\left.D>0.20 \mathrm{~h}^{-1}\right)$. Hence, when simulation studies do not employ experimentally determined $K_{\mathrm{s}}$ values, the results are limited to predicting trends.

\section{Ecological adaptations of facultatively methylotrophic bacteria}

When a bacterial specialist and a generalist have a common substrate, the specialist normally has the higher maximum specific growth rate (Gottschal et al., 1979; Dykhuizen \& Davies, 1980). It is anticipated that a specialist must have a higher $\mu_{\mathrm{m}}$ than a generalist if both are isolated from the same (variable) environment (Pianka, 1974). Hence, specialist, obligately methylotrophic bacteria are expected to exhibit higher maximum growth rates than generalist, facultatively methylotrophic bacteria. This is the case for the microbes studied here and thus, except at very low growth rates, obligate methylotroph B6/2 will outcompete and exclude facultative methylotroph DT26 from environments with a constant supply of methylamine. It seems that, in environments supplied continuously with methylamine and non- $\mathrm{C}_{1}$ organic compounds, facultatively methylotrophic bacteria are also outcompeted by methazotrophic bacteria, which use methylamine as nitrogen source only and non- $\mathrm{C}_{1}$ organic compounds as carbon source (J. D. Owens, unpublished observations).

In the absence of interactions other than competition, it is theoretically possible to have stable coexistence of two different organisms in a carbon- and energy-limited continuous culture supplied simultaneously with two different carbon substrates if each organism uses a unique substrate, or if one organism uses a unique substrate and the other uses both substrates (Stewart \& Levin, 1973). So, the observation here of the coexistence of an obligate methylotroph and a facultative methylotroph in a chemostat alternately supplied with methylamine and glucose does not, of itself, directly support the hypothesis that facultatively methylamine-using bacteria may be adapted to environments with an alternating nutrient supply. Nevertheless, the close similarity between the experimental and simulated cultures does lend some support to the hypothesis. To provide stronger evidence, experiments are required in systems with methazotrophic bacteria and non-methylamine-using heterotrophs in addition to the two 
methylotrophs. The fact that the predictions of the computer simulation model so closely mimic the laboratory data obtained in this work gives credence to the use of the simulation model to investigate the three- and four-organism systems. In view of the relative difficulty and long culture periods involved in these kinds of laboratory competition studies the possible use of computer simulations is a considerable benefit.

\section{Control of the composition of mixed microbial populations}

Over a range of dilution rates from 0.0625 to $0.20 \mathrm{~h}^{-1}$ facultative methylotroph DT26 and obligate methylotroph B6/2 coexisted in stable states in continuous cultures alternately supplied with methylamine and glucose/ammonium (Fig. 3). The relative proportion of each organism depended upon the imposed dilution rate. Hence, the use of an alternating nutrient supply may offer a practical approach to the control of the composition of defined, mixed populations.

This work was supported by a contract for research from the Ministry of Agriculture, Fisheries and Food. The results are the properties of the Ministry and are Crown Copyright.

\section{REFERENCES}

ANTHONY, C. (1982). The Biochemistry of Methylotrophs. London: Academic Press.

BADER, F. B. (1982). Kinetics of double-substrate limited growth. In Microbial Population Dynamics, pp. 1-32. Edited by M. J. Bazin. Boca Raton, Florida: CRC Press.

Baltzis, B. C. \& Fredrickson, A. G. (1983). Competition of two microbial populations for a single resource when one of them exhibits wall attachment. Biotechnology and Bioengineering 25, 2419-2439.

BICKNELl, B. (1978). Studies on bacteria utilizing methylamine as a sole nitrogen source. BSc thesis, Department of Microbiology, University of Sydney, Australia.

BiCKNELl, B. \& OWENS, J. D. (1980). Utilization of methyl amines as nitrogen sources by non-methylotrophs. Journal of General Microbiology 117, 89-96.

ChAO, L. \& RAMSDell, G. (1985). The effects of wall populations on coexistence of bacteria in the liquid phase of chemostat cultures. Journal of General Microbiology 131, 1229-1236.

Davison, B. H. \& Stephanopoulos, G. (1986). Effect of $\mathrm{pH}$ oscillations on a competing mixed culture. Biotechnology and Bioengineering 28, 1127-1137.

De Freitas, M. J. \& Fredrickson, A. G. (1978). Inhibition as a factor in the maintenance of the diversity of microbial ecosystems. Journal of General Microbiology 106, 307-320.

DYKhUIZEN, D. \& DAVIES, M. (1980). An experimental model: bacterial specialists and generalists competing in chemostats. Ecology 61, 1213-1227.

FREDRICKSON, A. G. (1977). Behaviour of mixed cultures of microorganisms. Annual Review of Microbiology 31, 63-87.

Gottschal, J. C. \& Thingstad, T. F. (1982). Mathematical description of two and three bacterial species under dual substrate limitation in the chemostat: a comparison with experimental data. Biotechnology and Bioengineering 24, 1403-1418.

Gottschal, J. C., De Vries, S. \& Kuenen, J. G. (1979). Competition between the facultatively chemolithotrophic Thiobacillus A2, an obligately chemolithotrophic Thiobacillus and a heterotrophic spirillum for inorganic and organic substrates. Archives of Microbiology 121, 241-249.

GotTschal, J. C., Pol, A. \& Kuenen, J. G. (1981 $a$ ). Metabolic flexibility of Thiobacillus A2 during substrate transitions in the chemostat. Archives of Microbiology 129, 23-28.

Gottschal, J. C., Nanninga, H. J. \& Kuenen, J. G. (1981b). Growth of Thiobacillus A2 under alternating growth conditions in the chemostat. Journal of General Microbiology 126, 85-96.

HARRISON, D. E. F. (1978). Mixed cultures in industrial fermentation processes. Advances in Applied Microbiology 24, 129-164.

HöFLE, M. G. (1983). Long-term changes in chemostat cultures of Cytophaga johnsonae. Applied and Environmental Microbiology 46, 1045-1053.

Ingraham, J. L., Maaløe, O. \& Neidhardt, F. C. (1983). Growth of the Bacterial Cell, p. 186. Sunderland, Mass.: Sinauier Associates.

Kemp, C. W., Robrish, S. A., CURTIS, M. A., Sharer, S. A. \& BOWEN, W. H. (1983). Application of a competition model to the growth of Streptococcus mutans and Streptococcus sanguis in binary continuous culture. Applied and Environmental Microbiology 45, 1277-1282.

Klei, H. E., Sundstrom, D. W. \& Molvar, A. E. (1975). Control of well-mixed biological reactors subject to variations in feed concentration and flow rate. Journal of Applied Chemistry and Biotechnology 25, 535-548.

Kuenen, J. G. \& GotTschal, J. C. (1982). Competition among chemolithotrophs and methylotrophs and their interactions with heterotrophic bacteria. In Microbial Interactions and Communities, vol. 1, pp. 153-187. Edited by A. T. Bull \& J. H. Slater. London: Academic Press.

LARGE, P. J. \& Green, J. (1984). Oxidation of mono-, di- and trimethylamine by methazotrophic yeasts: properties of the microsomal and peroxisomal enzymes involved and comparison with bacterial enzyme systems. In Microbial Growth on $C_{1}$ Compounds, pp. 155-164. Edited by R. L. Crawford \& R. S. Hanson. Washington, DC: American Society for Microbiology. 
LARSEN, V. F., Holdom, R. S., SPIVEy, M. J. \& TodD, M. (1978). Unit system for selection of mixed interactive cultures for industrial steady-state fermentations. In Techniques for the Study of Mixed Populations, pp. 179-211. Edited by D. W. Lovelock \& R. Davies. London: Academic Press.

LEGAN, J. D. (1985). Bacterial competition for methylamine under conditions of periodic nutrient alternation. PhD thesis, University of Reading, UK.

LegAN, J. D. \& OWENS, J. D. (1985). A note on the selection of a non-adhesive methylotrophic bacterium for use in continuous culture studies. Journal of Applied Bacteriology 58, 163-165.

LEGAN, J. D. \& OWENS, J. D. (1987). Determination of growth parameters of methylamine-using bacteria. Journal of General Microbiology 133, 1075-1080.

OWEns, J. D. \& Keddie, R. M. (1969). The nitrogen nutrition of soil and herbage coryneform bacteria. Journal of Applied Bacteriology 32, 338-347.

Pianka, E. R. (1974). Evolutionary Ecology. New York: Harper \& Row.

PicketT, A. M. (1982). Growth in a changing environment. In Microbial Population Dynamics, pp. 91-124. Edited by M. J. Bazin. Boca Raton, Florida: CRC Press.

Postgate, J. R. \& Hunter, J. R. (1964). Accelerated death of Aerobacter aerogenes starved in the presence of growth limiting substrates. Journal of General Microbiology 34, 459-473.

Ratnam, D. A., Pavlou, S. \& Fredrickson, A. G. (1982). Effects of attachment of bacteria to chemostat walls in a microbial predator prey relationship. Biotechnology and Bioengineering 24, 2675-2694.

Stewart, F. M. \& Levin, B. R. (1973). Partitioning of resources and the outcome of interspecific competition: a model and some general considerations. American Naturalist 107, 171-198.

STRAŠKbová, V. (1983). The effect of substrate shock on populations of starving bacteria. Journal of Applied Bacteriology 54, 217-224.

TURPIN, D. H. \& HARRISON, P. J. (1979). Limiting nutrient patchiness and its role in phytoplankton ecology. Journal of Experimental Marine Biology and Ecology 39, 151-166.

VAN GEMERDEN, H. (1974). Co-existence of organisms competing for the same substrates: an example among the purple sulfur bacteria. Microbial Ecology 1, 104-119.

ZATMAN, L. J. (1981). A search for patterns in methylotrophic pathways. In Microbial Growth on $C_{1}$ Compounds, pp. 42-54. Edited by H. Dalton. London: Heyden. 